\title{
Britain's best kept secret
}

\section{Die Enigma und die Funkaufklärung im Zweiten Weltkrieg ${ }^{1}$}

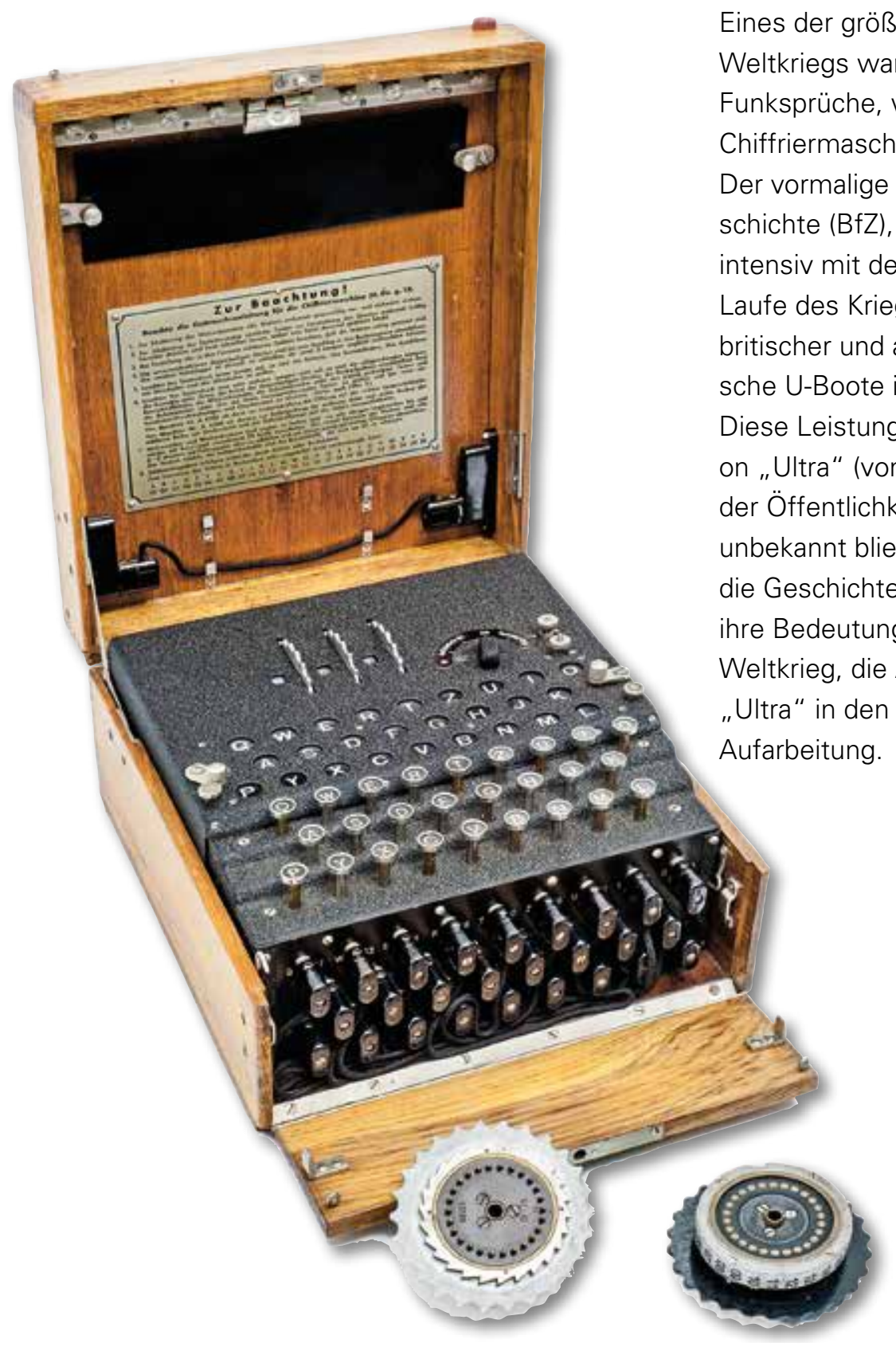

Abb. 1: Enigma mit Austauschwalzen (Foto: WLB / Larissa Arlt) 


\section{Der Krieg im Atlantik}

Großbritannien war während des Zweiten Weltkriegs stark auf die Versorgung über See angewiesen. Wie schon im Ersten Weltkrieg versuchte die deutsche Marine, Schiffe auf ihrem Weg nach Großbritannien zu attackieren, um so das Land von der Versorgung abzuschneiden. Da die deutsche Marine der britischen nicht gewachsen war, konnten solche Angriffe im Wesentlichen nur mit Hilfe von U-Booten erfolgen.

Zur Sicherung ihrer Transporte stellten die Briten Geleitzüge auf. Etliche Transportschiffe passierten gemeinsam den Atlantik und wurden dabei von Kriegsschiffen begleitet, die sie vor feindlichen U-Booten schützten. Als Gegenreaktion wurden deutsche U-Boote zu "Vorpostenstreifen“" zusammengezogen, um bestimmte Konvois abzufangen. Die Koordination der U-Boote erfolgte über Funk. Für eine erfolgreiche Kriegführung zur See war es unerlässlich, dass diese Funksprüche nicht abgehört werden konnten.

\section{Die Enigma}

\section{Geschichte und Funktionsweise}

Während des Ersten Weltkriegs hatten die Alliierten aus der Analyse des deutschen Funkverkehrs erhebliche strategische Vorteile gezogen. Vor dem Hintergrund dieser nach Kriegsende bekannt gewordenen kryptographischen Katastrophe suchte die deutsche Reichswehr nach einer sicheren Verschlüsselungsmethode für Funksprüche. Aufbauend auf älteren Patenten entwickelte Arthur Scherbius in den frühen 1920er Jahren eine Schlüsselmaschine, der er den Namen Enigma (griechisch „Rätsel“) gab. Die deutsche Reichsmarine erwarb 1926 eine eigens für das Militär modifizierte Version. Die 1935 von der Wehrmacht eingeführte Enigma wurde mit einigen Modifikationen bis Kriegsende 1945 verwendet.

Die Enigma ist weder eine Schreibmaschine noch ein Sender. Vielmehr handelt es sich um ein Anzeigegerät, das einen Text Buchstabe für Buchstabe in eine verschlüsselte Botschaft verwandelt, die auf Papier notiert und als Morse-Funkspruch versendet wurde. Ebenso kann mit dem Gerät eine verschlüsselte Botschaft wieder in den Klartext umgewandelt werden.

Die Enigma besteht aus folgenden Hauptbestandteilen: 1) eine Eingabetastatur, 2) mehrere rotieren- de Walzen, 3) eine nicht rotierende Umkehrwalze, 4) ein Steckerbrett sowie 5) ein Anzeigefeld. Die rotierenden Walzen bilden das Herzstück der Verschlüsselung.

Betätigt man eine der Eingabetasten (zum Beispiel das "S"), so fließt elektrischer Strom aus einer Batterie durch den Walzensatz und lässt ein Lämpchen mit einem anderen Buchstaben, der sogenannten Chiffre (in Abbildung 2 das "N"), aufleuchten. Bei der Dechiffrierung zeigen die Lämpchen dementsprechend Klartext an.

Damit Verschlüsselung und Entschlüsselung fehlerfrei vonstattengehen, müssen Sender und Empfänger dieselbe Ausgangsstellung der Walzen verwenden. Zunächst sind 3 Walzen aus einem Pool von 5 oder sogar 8 Walzen auszuwählen und in einer mit dem Empfänger abgestimmten Reihenfolge einzusetzen.

Danach sind die Walzen in eine ebenfalls abzustimmende Ausgangsstellung zu bringen. Auf den Walzen ist entweder das Alphabet mit 26 Buchstaben oder die den Buchstaben entsprechende Folge arabischer Zahlen von 01 bis 26 eingraviert. Jede

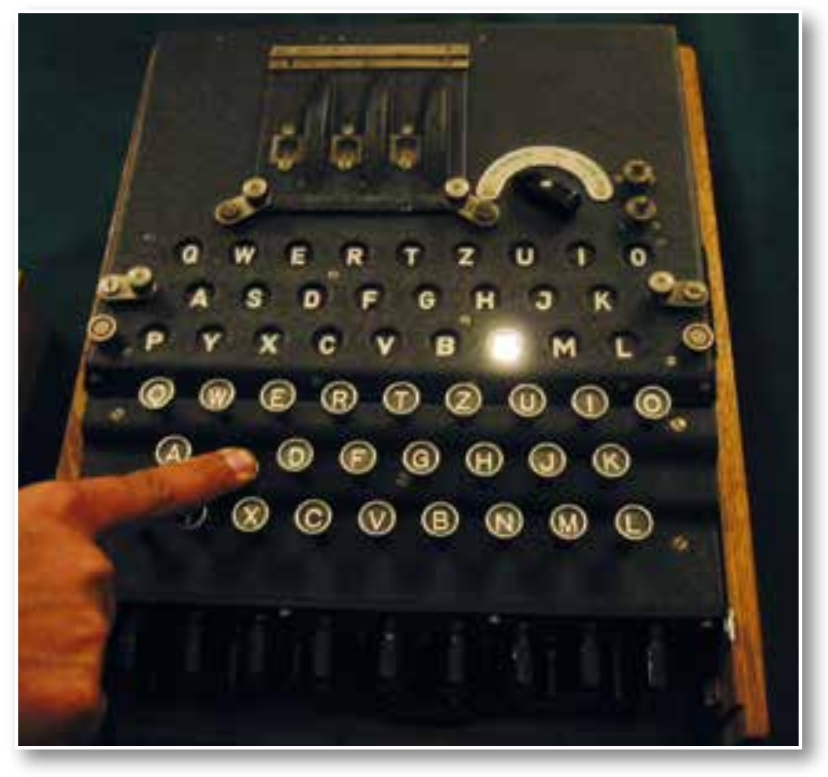

Abb. 2: Tastatur und Anzeigefeld der Enigma

Walze kann getrennt von den anderen an ihrem Außenring gedreht und in eine bestimmte Ausgangsposition gebracht werden.

Die Walzen sind in ihrem Inneren auf jeweils unterschiedliche Weise verdrahtet. Diese innere Verdrahtung kann nicht verändert werden. Das durch Anschlag einer Taste ausgelöste elektrische Signal wird von Walze zu Walze über Kontakte 
weitergeleitet. Eine am linken Ende der Walzen angebrachte sogenannte Umkehrwalze dreht sich nicht, vertauscht aber die Buchstaben und gibt das Signal an die Rotoren zurück. Das Signal tritt nun den Rückweg an. Durch die innere Verdrahtung der Walzen wird allerdings ein anderer Weg gewählt als auf dem Hinweg (Abb. 5).

Alle Walzen sind mechanisch miteinander verbunden. Die erste Walze dreht sich nach jedem Anschlag um einen Kontakt weiter. Wenn sich die erste Walze einmal komplett gedreht hat, dreht sich die zweite Walze um einen Kontakt weiter. Dieses Prinzip wird bei der dritten Walze fortgeführt. Ein vorn am Gerät angebrachtes Steckerbrett

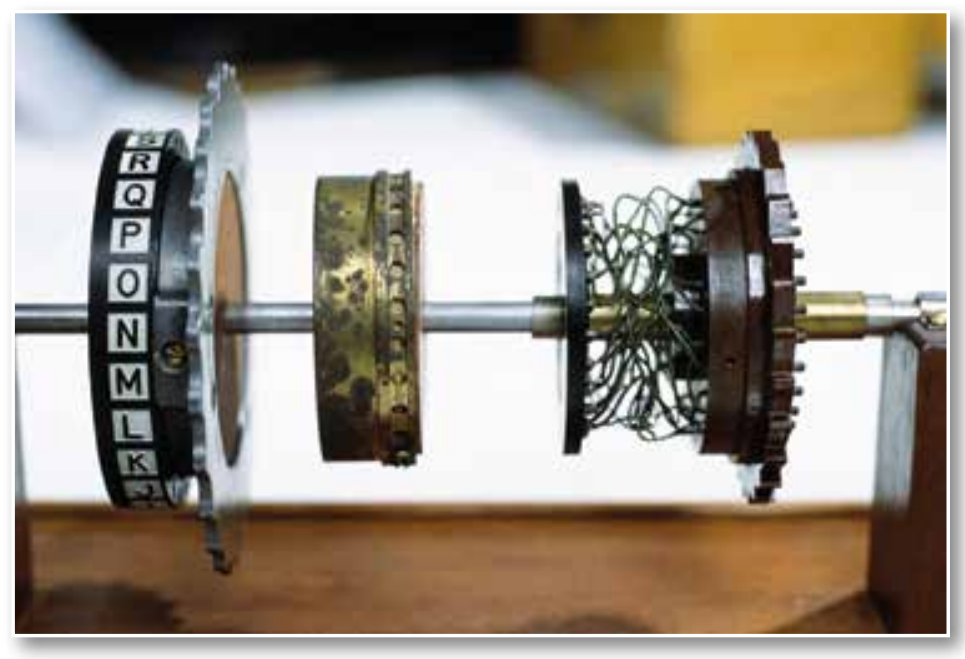

Abb. 3: Die innere Verdrahtung der Walzen (Bletchley Park Trust Ltd)
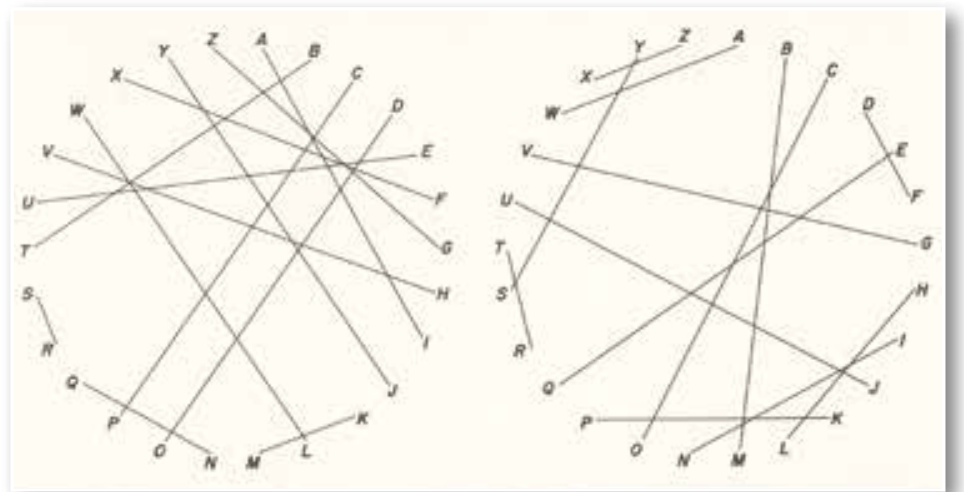

Abb. 4: Grafik der inneren Verdrahtung (WLB / Jürgen Rohwer)

dient dazu, die Variation des Buchstabentausches noch weiter zu erhöhen. Hier können durch Kabelverbindungen einzelne Buchstaben miteinander getauscht werden. So wird z.B. aus einem " $T$ " ein "U".

Die Gefahr der Entzifferung steigt, je mehr Text mit der gleichen Einstellung verschlüsselt wird. Die

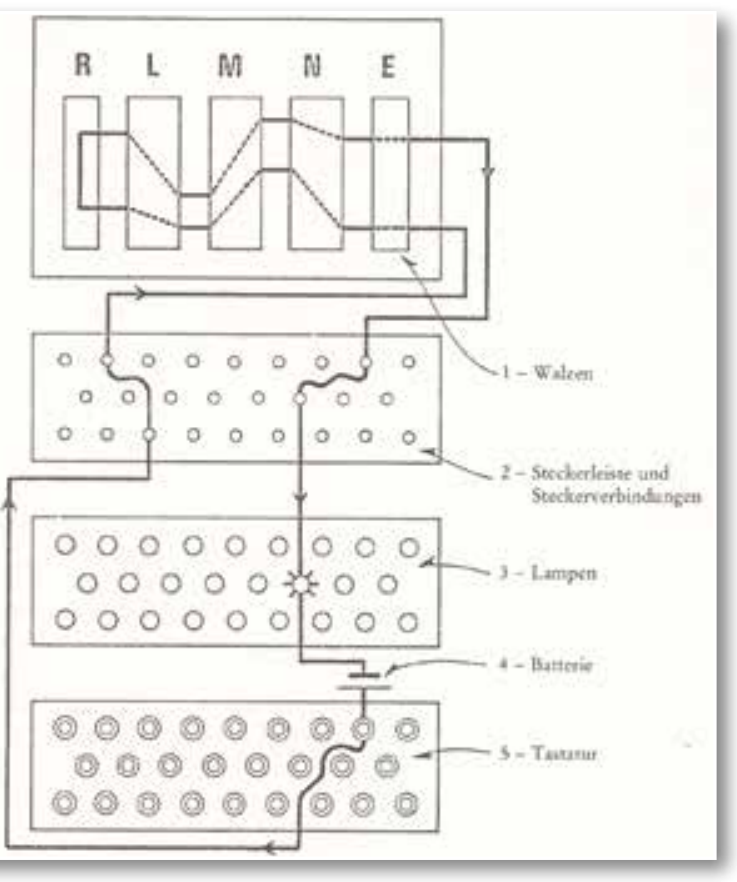

Abb. 5: Weg des elektrischen Impulses durch Steckerbrett und Walzen (WLB / Jürgen Rohwer)

Enigma sollte daher in kurzen Abständen immer wieder neu eingestellt und die Länge der Funksprüche auf 250 Zeichen begrenzt werden. Die Schlüsseleinstellung war in der Regel täglich, teilweise sogar mehrfach am Tag zu wechseln.

\section{Kryptographische Schwächen}

Die kryptographischen Stärken der Enigma sind im Wesentlichen durch den rotierenden Walzensatz gegeben. Durch die Drehung der Walzen wird erreicht, dass jeder Buchstabe des Textes mit einem neuen Alphabet verschlüsselt wird (polyalphabetische Verschlüsselung). Auf diese Weise sind klassische Angriffe zur Entzifferung des Geheimtextes, wie statistische Analysen oder Mustersuche, zum Scheitern verurteilt.

Mehrere Faktoren trugen dennoch dazu bei, dass die Enigma der deutschen Wehrmacht schon bei Kriegsbeginn keine ausreichende Sicherheit mehr bot. Eine entscheidende Rolle spielten "wahrscheinliche Wörter" (Cribs), deren Auftreten im Text zu erwarten ist. Dazu gehörten militärische Meldungen und Wetterbeobachtungen, die jeden Morgen pünktlich zur selben Zeit und vom selben Ort gesendet wurden. Sie waren häufig stereotyp abgefasst und enthielten viele Cribs wie z.B. MINENSPERRE oder GELEITZUG, die die britischen Codeknacker zur Entzifferung nutzten. Zuweilen provozierten die Briten bewusst Vorfälle, um Funksprüche mit vorhersehbarem Inhalt zu erhalten. 


\section{Die Entschlüsselung \\ Der polnische Geheimdienst}

Schon früh gab es Versuche, die Enigma zu entschlüsseln. In den 1920er Jahren gelangte der polnische Geheimdienst in den Besitz von Enigma-Maschinen, die als Zivilversionen im Handel käuflich waren. Die Militär-Enigma wurde häufig bei deutschen Manövern in Westpreußen eingesetzt. Viele chiffrierte Funksprüche wurden von den Polen aufgefangen und ermöglichten ihnen die Offenlegung der in militärischen Enigma-Versionen genutzten Walzen und ihrer inneren Verdrahtung. Es gelang den Polen sogar, eine Militärversion der Enigma nachzubauen.

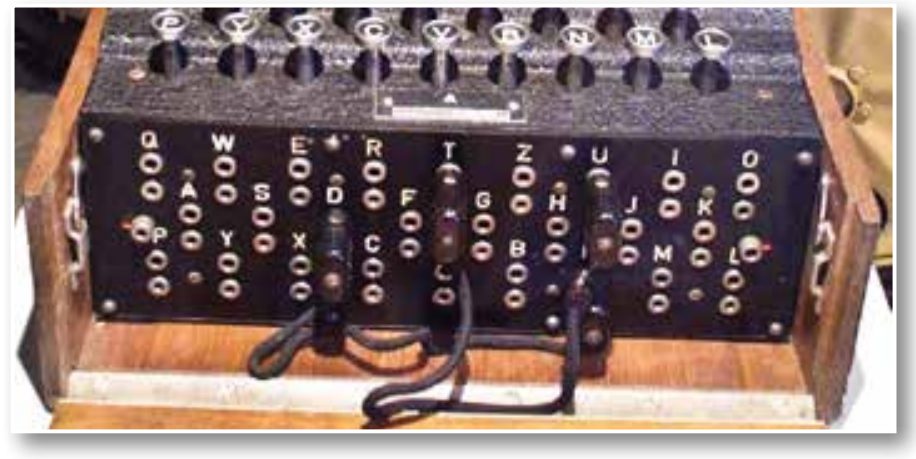

Abb. 6: Steckerbrett (https://commons.wikimedia.org/wiki/File:Enigma_ Verkehrshaus_Luzern.jpg)

1932 übergab Hans-Thilo Schmidt, ein unter dem Decknamen HE (Asché) für Frankreich arbeitender deutscher Spion, dem französischen Geheimdienstmitarbeiter und späteren Général Gustave Bertrand Schlüsseltafeln, eine Gebrauchsanweisung sowie Schlüsselunterlagen für die Enigma. Das Deuxième Bureau des französischen Geheimdienstes leitete die Unterlagen an britische und polnische Stellen weiter. Während es Franzosen und Briten nicht gelang, die Verschlüsselungsmechanismen zu durchschauen, glückte dem 27-jährigen polnischen Mathematiker Marian Rejewski 1932 der erste Einbruch in die Enigma.

Am Vorabend des Zweiten Weltkriegs gaben die Polen ihr Wissen an die verblüfften Briten und Franzosen weiter. Am 26. und 27. Juli 1939 präsentierten die polnischen Codeknacker bei einem Geheimtreffen im Wald von Pyry, knapp 20 km südöstlich von Warschau, Major Bertrand, dem französischen Kryptologen Henri Braquenié sowie britischen Geheimdienstmitarbeitern ihren EnigmaNachbau sowie von innen entwickelte kryptoanalytische Maschinen.

\section{Briten und Amerikaner}

Infolge des deutschen Überfalls auf Polen musste das Entschlüsselungszentrum im Pyry-Forst aufgelöst werden. Immerhin gelang den polnischen Kryptologen die Flucht vor der Wehrmacht und vor der Roten Armee. Namhafte Experten konnten sich über Rumänien nach Frankreich absetzen und unterstützten fortan die "Sektion Z“ im französischen Entzifferungszentrum Vignolles bei Paris.

Als deutsche Verbände 1940 auf Paris vorrückten, musste auch das Chateau de Vignolles blitzartig geräumt werden. Die dort vorhandenen EnigmaMaschinen sowie alle wichtigen Dokumente und Unterlagen wurden vernichtet; Bertrand und seine Equipe entkamen per Flugzeug nach Algier. Von nun an waren die Briten auf sich gestellt.

Die britischen Kryptoanalytiker begannen im etwa $70 \mathrm{~km}$ nordwestlich von London gelegenen Bletchley Park einen Angriff auf die Enigma. Zeitweilig waren bis zu 14.000 Frauen und Männer im Einsatz, um deutsche Funksprüche abzuhören und sie zu entschlüsseln.

Von entscheidender Bedeutung war die Zeit, die zum Knacken der Tagesschlüssel benötigt wurde. Der hochbegabte Mathematiker Alan Turing war der Meinung, dass diese Zeit nicht in Einzelverfahren, sondern nur mit Hilfe einer Maschine auf ein sinnvolles Maß reduziert werden konnte. Unter Hochdruck trieb er die Entwicklung eines analogen Rechners voran. Turings Idee war, durch ringförmige Verkettung von mehreren Enigma-Walzensätzen die praktisch unüberschaubare Anzahl von mehr als 200 Trilliarden Verschlüsselungsmöglichkeiten drastisch zu reduzieren. Die verbliebenen Kombinationen lief die so genannte "Turing-Bombe" dann durch, bis die korrekte Schlüsselstellung gefunden wurde.

Während der Einbruch in den Funkverkehr der Luftwaffe bereits am 22. Mai 1940 gelang, ließ die Entschlüsselung des Marine-Funkverkehrs zunächst auf sich warten. Die von der Marine verwendete Enigma wies Austausch-Walzen auf, deren innere Verdrahtung den Briten nicht bekannt war. Am 9. Mai 1941 kaperte der britische Zerstörer HMS Bulldog das deutsche U-Boot $\mathrm{U} 110$ und erbeutete eine intakte Marine-Enigma sowie sämtliche zu ihrer Verwendung notwendigen Geheimdokumente. Mit Hilfe dieses Coups gelang es den Briten noch im selben Monat, den deutschen Marine-Funkverkehr zu entschlüsseln. Der Erfolg währte nicht lange. 
1942 führte die Marine eine 4-Walzen-Enigma (,M4“) mit neuen Austauschwalzen ein. Für ein Jahr waren die Kryptologen in "Bletchley Park" nun faktisch "blind".

Nach Kriegseintritt der Vereinigten Staaten tauschten sich Engländer und Amerikaner über ihre Erfolge bei der Entschlüsselung des deutschen Funkverkehrs aus. Dadurch waren die Amerikaner in der Lage, die Turing-Bombe weiter zu verbessern. Ab April 1943 wurden mehr als 120 Exemplare einer Hochgeschwindigkeitsvariante der Turing-Bombe produziert, die speziell gegen die M4 gerichtet waren. Von nun an waren die deutschen U-Boote bis Kriegsende nicht mehr sicher.

\section{Deutsche Versäumnisse}

Die deutsche Seite war mehrheitlich so sehr von der Sicherheit der Enigma überzeugt, dass ein feindlicher Einbruch in ihr System kategorisch ausgeschlossen wurde. Da militärische Informationen im Krieg sehr schnell an Aktualität verlieren, galt es zudem als ausgemacht, dass eine etwaige Entzifferung zu viel Zeit in Anspruch nehmen würde, um effektive Gegenmaßnahmen einzuleiten. Dementsprechend wurde wenig zur Verbesserung der Maschine unternommen.

Mehrfach wurde die Schlüsselsicherheit der Enigma jedoch im Verlauf des Krieges in Frage gestellt, vor allem vom Befehlshaber der U-Boote, Karl Dönitz. Bereits kurz nach dem deutschen Einmarsch in Polen wurden die Erfolge der polnischen Entzifferungsabteilung bei der Rekonstruktion der EnigmaWalzen aufgedeckt. Vom Nachrichtendienst der Schweiz erhielt die Abwehrabteilung des Oberkommandos der Wehrmacht 1943 sogar einen direkten Hinweis auf die "Codeknacker" von Bletchley Park. Der deutsche Marinenachrichtendienst prüfte deshalb mehrfach die Sicherheit der Enigma, allerdings lediglich auf theoretischer Basis und auf Grund statistischer Erhebungen.

Erst ab Januar 1944 knackte ein Sachverständiger des deutschen Marinenachrichtendienstes auf empirischer Basis die Enigma und legte im Juni 1944 eine Liste der verheerendsten Fehler in ihrem Gebrauch vor. Zu diesem Zeitpunkt aber war es für Verbesserungen schon zu spät.

\section{Konsequenzen der Entzifferung}

Unstrittig ist, dass die Kompromittierung der Enigma von enormer strategischer und taktischer
Bedeutung für den Verlauf des Zweiten Weltkriegs war. Viele Historiker gehen davon aus, dass sich zwar ohne Entschlüsselung der Enigma am Ausgang des Krieges nichts geändert hätte. Der Krieg hätte aber vermutlich länger gedauert und hätte mehr Todesopfer gefordert.

Große Bedeutung gewann die Entschlüsselung des deutschen Funkverkehrs für die Schlacht im Atlantik. Mit Hilfe von "Ultra“ ließen sich Geleitzugschlachten nicht vollständig vermeiden, allerdings konnten bedrohte Konvois durch die genaue Kenntnis des Standorts der feindlichen U-Boote besser verstärkt werden.

Dank der amerikanischen Entschlüsselung gelang im Sommer 1943 die Versenkung von deutschen UTankern. Ohne diese Versorgungsfahrzeuge waren die deutschen U-Boote nicht in der Lage, sich im Atlantik zu halten und mussten nach Frankreich abgezogen werden. Der U-Boot-Krieg war für die Deutschen damit endgültig verloren.

Auch für den Erfolg der Landung in der Normandie am D-Day spielte „Ultra" eine wichtige Rolle. So wurde beispielsweise der Enigma-Schlüssel vom D-Day, also dem Tag der Landung der Alliierten in der Normandie, durch den Crib „WETTERVORHERSAGEBISKAYA", den die britischen Kryptoanalytiker leicht erraten konnten und korrekt vermuteten, in weniger als zwei Stunden gebrochen.

\section{Ein lang gehütetes Geheimnis}

Das durch die erfolgreiche Funkaufklärung gewonnene Wissen nutzten die Alliierten sehr behutsam, um den Deutschen keine Anhaltspunkte dafür zu geben, dass sie die Enigma entschlüsselt hatten. Es galt zu verhindern, dass die Deutschen auf andere Verschlüsselungsformen auswichen. So waren die über "Ultra" informierten Befehlshaber gehalten, ihre Operationsbefehle stets mit einer glaubwürdig erscheinenden "cover story" auszustatten, was die Herkunft der zugrundeliegenden Informationen anging. Diese Verschleierungstaktik war so erfolgreich, dass die Existenz und die Leistungen von „Ultra" den Deutschen vollkommen verborgen blieben. Auch nach Kriegsende behielten Briten und Amerikaner das Geheimnis für sich, um es eventuell weiterhin militärisch nutzen zu können. So blieb die Entzifferung ein gut gehütetes Geheimnis, und selbst Experten gingen lange Zeit davon aus, dass die Enigma weitgehend einbruchsicher gewesen sei. 


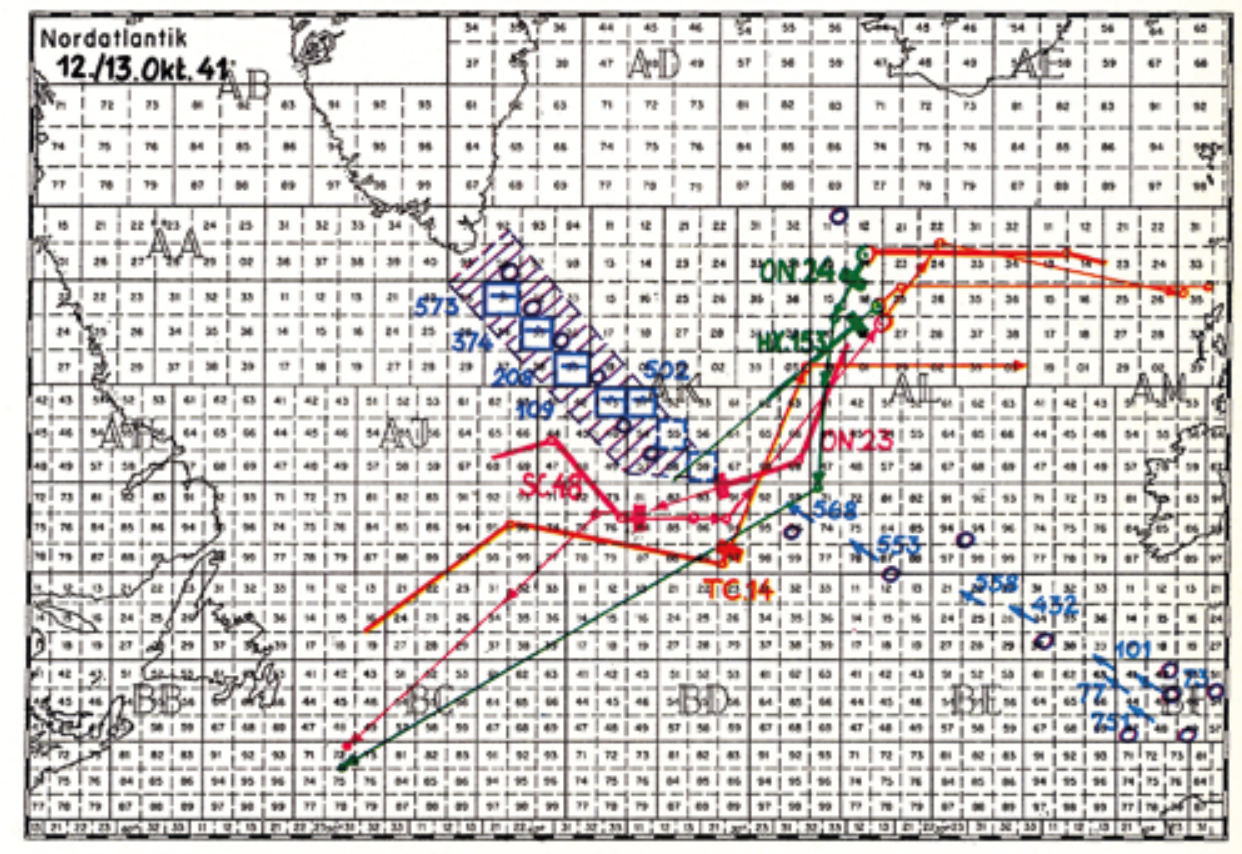

Abb. 7: Grafik zu Geleitzugschlachten im Atlantik. Aufstellung eines U-Boot-Abfangstreifens und die Umlenkung der alliierten Konvois (WLB / Jürgen Rohwer)

\section{Die Dokumentation des U-Bootkriegs in der Bibliothek für Zeitgeschichte (BfZ)}

1959 wurde Jürgen Rohwer Direktor der BfZ in Stuttgart, einer wichtigen Anlaufstelle für die Forschung zum Zweiten Weltkrieg. Rohwer pflegte ein leidenschaftliches Interesse für die Marinegeschichte. Nach dem Krieg unterstützte er die Arbeit des Naval Historical Teams, einer Gruppe deutscher Offiziere, die im britischen und amerikanischen Auftrag die Operationen der deutschen Kriegsmarine aufarbeitete. Wo immer es inm möglich war, sammelte er Informationen zu seinem besonderen Interessegebiet, dem deutschen U-Boot-Krieg und seinen "Erfolgen“ bei der Versenkung alliierter Schiffe. Unter anderem tippte er heimlich das Kriegstagebuch des Befehlshabers der U-Boote, Karl Dönitz, ab. Dönitz, der auch Oberbefehlshaber der Kriegsmarine und Nachfolger Hitlers als deutscher Reichspräsident gewesen war, lernte Rohwer nach dessen Haftentlassung auch persönlich kennen und knüpfte einen engen Kontakt zu inm.

1957 bis 1986 war Rohwer Hauptschriftleiter der bedeutenden Fachzeitschrift "Marine-Rundschau". In dieser Funktion baute er in der BfZ eine umfangreiche Sammlung zur Marinegeschichte auf, die unter anderem eine halbe Millionen Fotos umfasst. Außerdem beteiligte sich Rohwer mit zahlreichen
Publikationen an der Forschung zum Seekrieg und zu den Geheimdiensten im Zweiten Weltkrieg.

Mit Hilfe eines großen Korpus an Primärquellen (U-Boot-Schussmeldungen, Kriegstagebuch des Befehlshabers der U-Boote, Schiffsverlustliste der britischen Admiralität und weitere nationale Schiffsverlustlisten) schuf Rohwer eine Kartei mit mehr als 18.000 Karten, die sämtliche Torpedoschüsse deutscher U-Boote im Zweiten Weltkrieg dokumentiert. Diese Kartei wertete er 1968 in seinem Buch "U-Boot-Erfolge der Achsenmächte" aus. Die Analyse der Daten zeigte, dass die Versenkung alliierten Schiffsraums im Atlantik ab Mai 1941 wesentlich niedriger ausgefallen war, als die eingesetzte Zahl an U-Booten vermuten ließ. Für das Jahr 1943 kam Rohwer zu einem ähnlichen Ergebnis.

Angesichts dieser Befunde stellte sich Rohwer die Frage, ob die Verschlüsselung der deutschen Marine-Funksprüche während des Krieges sicher gewesen sei. Er befragte wichtige Zeitzeugen, doch diese hielten eisern dicht und erwähnten das noch immer geheime Projekt "Ultra" mit keinem Wort. So hatte Stephen Roskill, Verfasser der offiziellen britischen Seekriegsgeschichte, 1959 in einem Buch publik gemacht, dass die Briten 1941 bei der Aufbringung des U-Boots U110 wichtige Schlüsselunterlagen zur Enigma erbeutet hatten. Doch auch unter Einsatz mehrerer Flaschen Wein gelang es Rohwer nicht, Roskill zu entlocken, ob es den Briten mit Hilfe dieser Unterlagen gelungen war, die Enigma zu knacken. So ging Rohwer wie andere Historiker davon aus, dass Funkpeilung von Land und "High Frequency Direction Finder" auf Geleitfahrzeugen die Ursache für die zunehmenden Misserfolge im deutschen U-Boot-Krieg gewesen seien. 


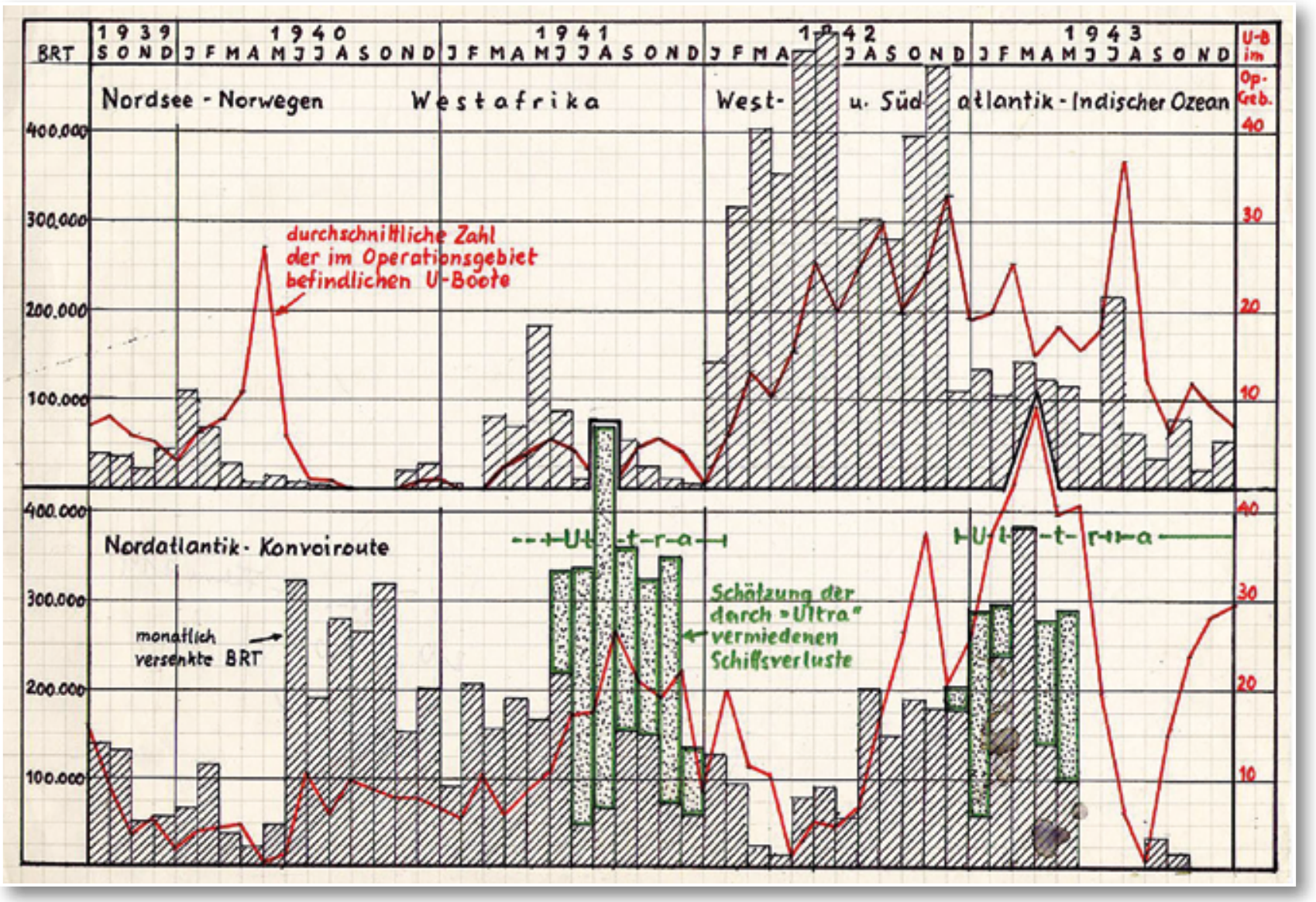

Abb. 8: Monatliche Versenkungsziffern der deutschen U-Boote 1939-1943 (WLB / Jürgen Rohwer)

\section{Die sensationelle Enthüllung}

Im Herbst 1974 kam es zu einer historischen Sensation: Der britische Luftwaffenoberst Frederick W. Winterbotham enthüllte mit seinem Buch "The Ultra Secret" das bis dahin "bestgehütete Geheimnis des Zweiten Weltkrieges". 1976 gaben die britische und die amerikanische Regierung außerdem die „Ultra“-Akten für die wissenschaftliche Nutzung frei.

Es folgte eine intensive wissenschaftliche Auseinandersetzung mit dem Thema, an der sich auch Rohwer beteiligte. 1978 organisierte er einen großen internationalen Kongress zur Funkaufklärung im Zweiten Weltkrieg in Bonn und Stuttgart. Auf der Tagung trafen wichtige Zeitzeugen und Historiker aus der Bundesrepublik Deutschland, England, den USA, Kanada, Polen, Frankreich, Finnland und der Schweiz zusammen, um über die Bedeutung von „Ultra“ zu diskutieren. Die Vorträge und Diskussionen wurden 1979 in dem Buch „Die Funkaufklärung und ihre Rolle im Zweiten Weltkrieg" veröffentlicht.
Auch in der Folgezeit beschäftigte sich Rohwer mit der Funkaufklärung und nachrichtendienstlichen Tätigkeiten. In Anerkennung seiner Rolle in der Forschung wurde er von 1985 bis 2000 Vizepräsident der Commission Internationale d'Histoire Militaire und von 1993 bis 1999 Chairman der neu gegründeten International Intelligence History Association.

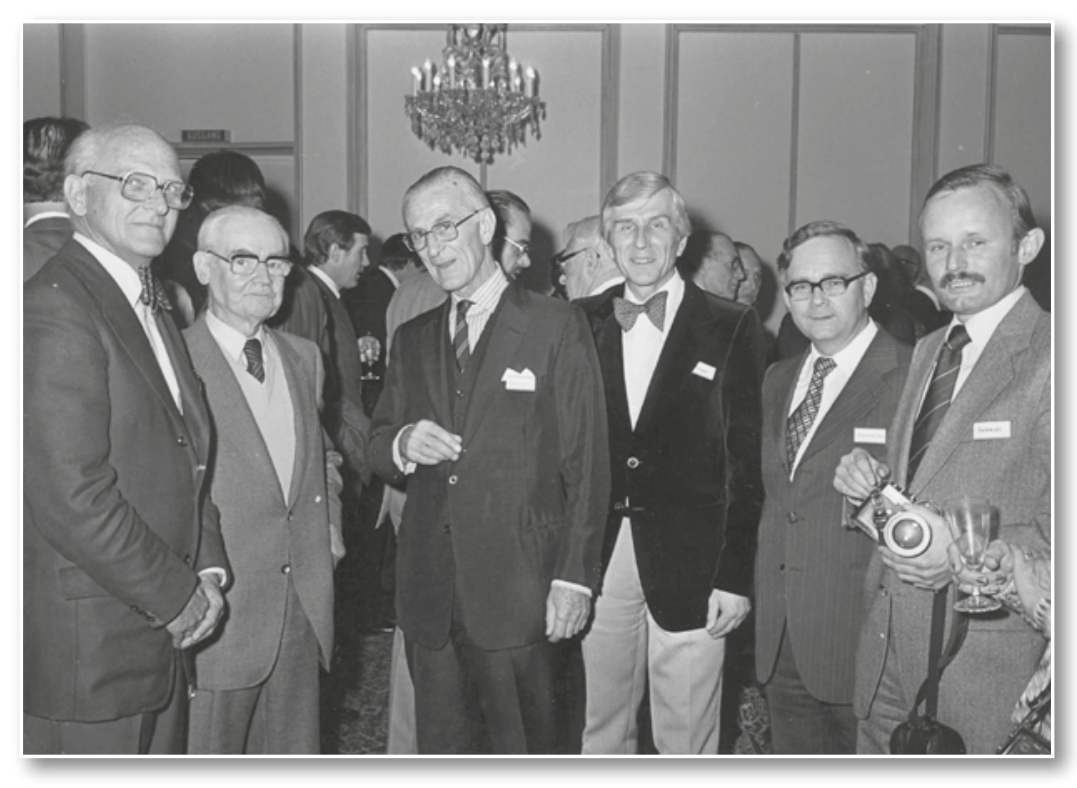

Abb. 9: Internationales Symposium zur Funkaufklärung im Zweiten Weltkrieg, Bonn und Stuttgart 1978. Dritter von rechts: Jürgen Rohwer (WLB / Foto: Gerhard Klein) 
Rohwer verstarb 2015 im Alter von 91 Jahren. 1978 zeigte die BfZ begleitend zur Tagung in Stuttgart eine Ausstellung zur Funkaufklärung im Zweiten Weltkrieg. Für diese Ausstellung wurde eigens ein Exemplar der Enigma angeschafft. Dieses Exemplar wurde auch in der Ausstellung „100
Jahre Bibliothek für Zeitgeschichte. 1915-2015“ gezeigt, die vom 20. November 2015 bis 9. April 2016 in der Württembergischen Landesbibliothek zu sehen war.

Christian Westerhoff / Thomas Weis

\section{Literatur}

Bertrand, Gustave: Énigma ou la plus grande énigme de la guerre 1939-1945, Paris 1973.

Erskine, Ralph: Enigma's Security. What the Germans really knew, in: Michael Smith / Ralph Erskine (Hg.): Action this day, London 2001, S. 370-385.

Rohwer, Jürgen: Der Einfluß der alliierten Funkaufklärung auf den Verlauf des Zweiten Weltkrieges, in: Vierteljahrshefte für Zeitgeschichte, 27. 1979, H. 3, S. 325-369.

Rohwer, Jürgen: Die U-Boot-Erfolge der Achsenmächte 19391945, München 1968.

Rohwer, Jürgen: Vom Naval Historical Team zum Arbeitskreis für Wehrforschung, in: Hartmut Klüver / Thomas Weis (Hg.):
Marinegeschichte - Seekrieg - Funkaufklärung. Festschrift für Jürgen Rohwer, Düsseldorf 2004, S. 79-88.

Rohwer, Jürgen / Jäckel, Eberhard (Hg.): Die Funkaufklärung und ihre Rolle im Zweiten Weltkrieg, Stuttgart 1979.

Roskill, Stephen Wentworth: The secret Capture, London 1959.

Weis, Thomas: Jürgen Rohwer, die Bibliothek für Zeitgeschichte und das Marinearchiv im Kalten Krieg, in: Christian Westerhoff (Hg.): 100 Jahre Bibliothek für Zeitgeschichte. 1915-2015, Stuttgart 2015, S. 108-127.

Winterbotham, Frederick William: The Ultra Secret, London 1974. 